\title{
Cardiovascular risk and BDNF concentration in vegetarians in the city of São Paulo - SP
}

\begin{abstract}
Cardiovascular diseases, main causes of death in the world, have their etiology related to a series of risk factors. Currently, there is a massive effort in the reduction of risks of these diseases, most especially, through food. In this sense, vegetarian diet can play an important role, by adjusting important biochemical parameters related to such diseases. Meanwhile, studies that contemplate the role of vegetarian diet in the adjustment of risk factors for cardiovascular diseases especially from biomolecular parameters are still insufficient. The objective of this study was to evaluate the relation between cardiovascular risk and the concentration of the Brain-Derived Neurotrophic Factor (BDNF) in vegetarians as compared to omnivores.
\end{abstract}

Methods: It is a cross-sectional study with 96 subjects (56 vegetarians and 40 omnivores). Nutritional status (weight, waist circumference and body mass index), biochemical parameters (fasting blood glucose, insulin, total cholesterol and fractions) and biomolecular profile (BDNF) were evaluated.

Results: As compared with omnivores, vegetarians present lower body weight $(\mathrm{p}=0.032)$, lower BMI $(p<0.001)$, lower WC values $(p=0.003)$, lower blood glucose $(p=0.004)$ and HOMA-IR. Homeostatic model assessment - insulin resistance $(\mathrm{p}=0.021)$ and higher HDL-c concentration $(\mathrm{p}=0.008)$. Regarding cardiovascular risk, vegetarians have lower Castelli Risk Indexes $1(\mathrm{p}=0.001)$, as well as 17 times more probability of obtaining the index within normal values.

Discussion: There was no difference between the BDNF means between vegetarians and omnivores; however, subjects with higher BDNF concentrations have higher risk of presenting an HDL-c reduction.

Keywords: brain-derived neurotrophic factor, cardiovascular risk, vegetarian diet, nutritional status, biochemical parameters
Volume 12 Issue 6 - 2019

\author{
Carolina Vieira de Mello Barros Pimentel,' \\ Elizabeth Teodorov ${ }^{2}$ Viviane Lazari \\ Simomura, ${ }^{3}$ Marcelo Macedo Rogero, ${ }^{4}$ Sonia \\ Tucunduva Philippi ${ }^{4}$ \\ Institute of Health Sciences, Universidade Paulista, Brazil \\ ${ }^{2} \mathrm{ABC}$ Federal University, Santo André, Brazil \\ ${ }^{3}$ Community University of Chapecó Region, Brazil \\ ${ }^{4}$ Department of Nutrition, University of São Paulo - USP, Brazil
}

Correspondence: Carolina Vieira de Mello Barros Pimentel, Institute of Health Sciences.ICS, Paulista University, University of São Paulo - UNIP, Sao Paulo, Brazil, Tel 55 | | 983476837 ,

Email cpimentel@carolinapimentel.com.br

Received: October 17, 2019 | Published: November 04, 2019
Abbreviations: BDNF, Brain-Derived Neurotrophic Factor; CVD, cardiovascular diseases; LDL-c, low-density lipoprotein; HDL-c, high density lipoprotein; DM, diabetes mellitus; TC, total cholesterol; TG, triglycerides; BMI, body mass index; WC, waist circunference

\section{Introduction}

Cardiovascular diseases (CVD) are the main causes of death in Brazil, which account for about $20 \%$ of all deaths in individuals above 30 years of age ${ }^{1}$ and, although progressive reduction in mortality has been observed in Brazil, rates continue to be high. ${ }^{2}$ Within the Brazilian context, chronic diseases accounted for $70 \%$ of deaths in the country, where cardiovascular disease is the main group of those diseases. ${ }^{3}$ In this sense, the importance of chronic diseases as cause of death intensifies the importance of strategies that aim at the reduction of their risk and their risk factors. ${ }^{3}$

The likelihood of CVD occurrence increases in the presence of atherosclerosis-associated risk factors, such as, high levels of lowdensity lipoprotein (LDL-c). ${ }^{4}$ In addition to these factors, Diabetes Mellitus (DM) also contributes to an increase in Brazilian morbidity and high prevalence of overweight and obesity and consumption below the recommended value in relation to intake of fruits and vegetables sum up to this scenario. ${ }^{5}$

For the last 20years, epidemiological studies have documented important and significant benefits of vegetarianism in reducing the risk of CVD, Diabetes Mellitus, obesity, hypertension and cancer, ${ }^{6-9}$ as well as in the influence of mortality. ${ }^{10,11}$ Studies with Brazilian vegetarians have shown reduced values of total cholesterol (TC), LDL-c and triacylglycerols (also known as triglycerides, TG). ${ }^{12,13}$

The effects of the vegetarian diet on health should be understood beyond the mere suppression of the intake of meat products, ${ }^{14}$ since this diet promotes an increase in the intake of fiber, polyunsaturated fatty acids, vitamins, minerals and phytochemicals, and it has lower caloric content and lower amount of saturated fat. ${ }^{15-17}$ Regarding the understanding of the effects of vegetarian diet standards to health, it is important to evaluate molecular pathways that modulate possible outcomes in heath, such as the Brain-Derived Neurotrophic Factor (BDNF) expression and the vulnerability of the atheroma plaque.

BDNF is a basic protein, a member of the neurotrophin family, and which plays an essential role in neurobiology, as it positively interferes in brain neuroplasticity and, in particular, in neural survival, 
differentiation and proliferation. ${ }^{18-20}$ In addition, BDNF influences learning, memory, mood and stress responsiveness. ${ }^{21}$ it is the most widely distributed neurotrophin in the central nervous system, and it has the ability of regulating various biological functions. ${ }^{20}$

Most recently, the endocrine role and its relation with cardiovascular diseases, which also seems to be associated to nutritional factors, as studies showed that circulating levels of BDNF are related with the regulation of energetic metabolism and CVD risk factors, such as positive correlation between circulating levels of BDNF and body mass index (BMI), TC, TG and LDL-c..22-24

Generally speaking, there are few studies focusing on the vegetarian diet, particularly in relation to its possible modulating role in biomolecular parameters. Studies that relate BDNF plasma levels and cardiovascular endpoints can contribute towards a better understanding of the mechanism of action of this neurotrophic factor and, thus, they can be the basis of therapies that modify the circulating levels of this protein, with the objective of promoting health, as for example, weight and plasma lipoproteins management. ${ }^{25-29}$ The objective of this study was to evaluate the relation between cardiovascular risk and the BDNF expression in vegetarians as compared to omnivores.

\section{Material and methods}

This is a cross-sectional study. At the end, 96 subjects (56 vegetarians and 40 omnivores) were included in the study. The first phase of data collection consisted in the explanation about the research, application of the informed consent form, application of the questionnaire containing sociodemographic information, type of diet, lifestyle and evaluation of nutritional status, with measurement of weight, height and waist circumference. The second phase consisted in the collection of blood samples for analysis of biochemical and molecular parameters.

\section{Type of diet}

In order to classify the subjects in relation to the alimentary practice, it was first questioned whether the subject considered himself/herself a semi-vegetarian, ovo-lacto vegetarian, lacto vegetarian, vegan or omnivore, classified according to the definitions provided by the Brazilian Vegetarian Society.

\section{Nutritional status variables}

It was evaluated by means of BMI, obtained by dividing body weight $(\mathrm{kg})$ by the height squared $\left(\mathrm{m}^{2}\right)$. To obtain the weight, the subjects were positioned at the center of the electronic scale (Welmy ${ }^{\circledR}$, model R-110). The height was measured using a portable anthropometer (Estad ${ }^{\circledR}$ - Alturexata, $0.35 \mathrm{~m}$ to $\left.2.13 \mathrm{~m}\right)$. The adults were weighed and measured with minimal dressing and barefooted. ${ }^{30}$ The height of each participant was measured by positioning him/her with his/her back against a vertical platform, on a flat surface, with the same clothes for weight measurement, heels together and the head positioned so that the line of sight remains perpendicular to the body (Frankfurt plane). ${ }^{30}$

The waist circumference (WC) was used to evaluate the body composition, and the standard of measurement in the umbilical scar was adopted, by using an inextensible measuring tape with a Sanny ${ }^{\circledR}$ lock. The classification of the nutritional status in accordance with the BMI was based on the cut-off points recommended by the
World Health Organization ${ }^{30}$ and classified as normal $\left(18.5 \mathrm{~kg} / \mathrm{m}^{2}\right.$ to $\left.24.9 \mathrm{~kg} / \mathrm{m}^{2}\right)$ and altered $\left(>24.9 \mathrm{~kg} / \mathrm{m}^{2}\right)$. The WC was also classified in accordance with the risk of metabolic complications associated to obesity, (normal for women when $\leq 80 \mathrm{~cm}$ and altered or at risk if $>80 \mathrm{~cm}$; for men, the WC considered normal was $\leq 94 \mathrm{~cm}$ and altered or at risk $>94 \mathrm{~cm}) .{ }^{31}$

\section{Biochemical variables associated to cardiovascular risk}

For obtainment of biochemical parameters, the following tests were performed: blood glucose (Automated Enzymatic Colorimetric); insulin (Chemiluminescent Microparticle Immunoassay - CMIA ${ }^{\circledR}$ ); $\mathrm{TC}$ and fractions (Automated Enzymatic). All procedures were conducted in a clinical analysis laboratory.

For the analysis, the biochemical variables were classified into normal and altered, by using the following reference ranges: altered fasting blood glucose $(\geq 110 \mathrm{mg} / \mathrm{dL})$, altered TC $(\geq 200 \mathrm{mg} / \mathrm{dL})$, altered LDL-c $(\geq 100 \mathrm{mg} / \mathrm{dL})$, altered high density lipoprotein (HDL-c) $(\leq$ $40 \mathrm{mg} / \mathrm{dL}$ for men and $\leq 50 \mathrm{mg} / \mathrm{dL}$ for women) and altered TG $(\geq 150$ $\mathrm{mg} / \mathrm{dL}$ ). The Castelli Risk Index 1 - TC/HDL-c Ratio, used to predict the risk of ischemic coronary disease, was also calculated. Values above 4.4 for females and 5.1 for males were considered altered.

\section{BDNF plasma levels}

For plasma analysis of BDNF, blood collection was performed in tubes with EDTA anticoagulant and centrifuged for 30minutes, at $6,000 \mathrm{rpm}$ at $8{ }^{\circ} \mathrm{C}$. BDNF levels were evaluated with BDNF Emax ${ }^{\circledR}$ ImmunoAssay System (Promega ${ }^{\circledR}$, Madison, WI, USA), in accordance with the manufacturer's specifications.

\section{Statistical Analysis}

All analyses were performed using the SPSS software for Windows version 20.0. For the analysis of the results the characterization of nutritional status, biochemical and molecular analyses were performed by means of proportions, means \pm standard deviations (SD).

The Kolmogorov-Smirnov test was conducted to analyze the variables normality. The difference between the proportions was tested through the chi-squared test (level of significance $\mathrm{p}<0.05$ ). The means were compared using Student's t test, when the distribution of the variable could be estimated by the normal distribution or, otherwise, by the Mann-Whitney U test. The assessment of aspects that predisposed cardiovascular risk was elaborated through the following steps:

1. Univariate models were constructed, and the variables that showed association with the groups (altered Castelli $1 \mathrm{x}$ Normal) $(\mathrm{p}<0.05)$ through the chi-squared test or, when continuous, through the Student's t test and Mann-Whitney U test, were eligible.

2. The BDNF values were divided into two percentiles, using the median as cut-off point; thus, the analyses of association with other categorical variables were performed, using the chi-square test.

\section{Ethical aspects}

The present study is approved by the Research Ethics Committee (REC), of research protocol No. 2260 of the Faculty of Public Health, University of São Paulo. 


\section{Results}

\section{Characterization of vegetarians and omnivores}

Table 1 compares the biochemical and anthropometric results that may have relation between cardiovascular risk and the plasma levels of BDNF between vegetarians and omnivores; as it can be observed; there was no statistically significant difference for age.

Table I Comparison of anthropometric and biochemical variables between vegetarian and omnivore groups, São Paulo, Brazil, 2014

\begin{tabular}{|c|c|c|c|}
\hline Variables & $\begin{array}{l}\text { Vegetarians } \\
\text { Mean士SD }\end{array}$ & $\begin{array}{l}\text { Omnivores } \\
\text { Mean士SD }\end{array}$ & p Value \\
\hline Age(years) & $33.8 \pm 7.6$ & $33.2 \pm 6,9$ & 0.688 \\
\hline Weight(kg) & $63.9 \pm 10.4$ & $69.4 \pm 14,6$ & $0.032 * a$ \\
\hline $\mathrm{WC}(\mathrm{cm})$ & $81.8 \pm 8.2$ & $87.8 \pm 10,9$ & $0.003^{* a}$ \\
\hline $\mathrm{BMI}\left(\mathrm{kg} / \mathrm{m}^{2}\right)$ & $22.5 \pm 2.6$ & $25.0 \pm 3,9$ & $0.000 * \mathrm{a}$ \\
\hline Blood Glucose(mg/dl) & $82.13 \pm 9.11$ & $85.8 \pm 10,87$ & $0.004^{* b}$ \\
\hline Insulin(Uu/ml) & $5.69 \pm 3.00$ & $6.89 \pm 3,16$ & 0.061 \\
\hline HOMA-IR & $\mathrm{I} .17 \pm 0.70$ & $1.48 \pm 0.8 \mathrm{I}$ & $0.021 * \mathrm{~b}$ \\
\hline $\mathrm{TC}(\mathrm{mg} / \mathrm{dL})$ & $170.45 \pm 35.80$ & $175.95 \pm 33.46$ & 0.447 \\
\hline $\mathrm{HDL}(\mathrm{mg} / \mathrm{dL})$ & $54.88 \pm \mid 4.44$ & $47.30 \pm 12.27$ & $0.008^{* a}$ \\
\hline LDL(mg/dL) & $99.82 \pm 26.86$ & $108.59 \pm 28.4$ & 0.127 \\
\hline TG(mg/dL) & $84.11 \pm 39.26$ & $100.33 \pm 54.24$ & 0.100 \\
\hline Castelli Risk Index I & $3.23 \pm 0.84$ & $3.90 \pm 0.99$ & $0.00 I^{* a}$ \\
\hline
\end{tabular}

$* 5 \%$ level of significance $(p<0.05)$

a. Student t- b. Mann-Whitney

In relation to the nutritional status, vegetarians present lower body weight $(\mathrm{p}=0.032)$, lower BMI $(\mathrm{p}<0.001)$ and lower WC values $(\mathrm{p}=0.003)$. As compared with omnivores, vegetarians present lower blood glucose $(p=0.004)$ and HOMA-IR $(p=0.021)$, and they also present lower Castelli risk indexes $1(\mathrm{p}=0.001)$ and higher HCL-c concentration $(\mathrm{p}=0.008)$. There was no difference for plasma concentrations of TC, TG and LDL-c.

\section{Cardiovascular risk between vegetarians and omnivores}

Although there is statistically significant difference between the anthropometric and biochemical variables associated to the nutritional status, it can be observed that the mean values found for BMI, blood glucose and HDL-c are within the desirable parameters. As seen in Table 1, vegetarians have lower body weight, lower BMI and WC values, as well as normal biochemical parameters, which infers in the greater probability of presenting Castelli Risk Index values within normality.

\section{Plasma concentration of BDNF}

Regarding plasma concentration of BDNF between vegetarians and omnivores, vegetarians were found to have the lowest plasma BDNF averages, and there was no difference between the means of this neurotrophic factors between vegetarians and omnivores $(662.8 \pm 276.5 \mathrm{pg} / \mathrm{ml}$ vs. $698.1 \pm 314.9 \mathrm{pg} / \mathrm{ml} \mathrm{p}=0.563)$. In addition, the gender-stratified analysis showed that vegetarian women had lower BDNF plasma concentrations when compared to omnivores women $(658.64 \pm 296.29 \mathrm{pg} / \mathrm{ml}$ vs. $705.56 \pm 317.00 \mathrm{pg} / \mathrm{ml})$ and, among men, this same pattern was observed, with lower BDNF plasma concentration among vegetarians in relation to omnivores $(668.81 \pm 251.82 \mathrm{pg} / \mathrm{ml}$ vs. $682.49 \pm 322.65 \mathrm{pg} / \mathrm{ml})$.

To increase the power of the sample, the BDNF plasma concentration was stratified in accordance with the median $(626.0 \mathrm{pg} /$ ml) (Table 3).

Still with regard to risk factors for cardiovascular diseases and the plasma concentration of BDNF, it was possible to observe (Table 4) a statistically significant difference for the plasma concentration of BDNF, according to the HDL-c parameters. Subjects with a BDNF plasma concentration above the median $(626.0 \mathrm{pg} / \mathrm{mL})$ have $66 \%$ probability of presenting HDL-c values below those desirable (altered), while in those with BDNF values up to $626.0 \mathrm{pg} / \mathrm{ml}$ there is a reduction in this probability to $43 \%$. It can then be inferred that subjects with higher plasma concentrations of BDNF have a $52 \%$ risk of having altered HDL-c (below the desired values).

Table 2 Comparison of the study's categorical variables between the groups that have normal and altered Castelli risk index I. São Paulo, Brazil, 2014

\begin{tabular}{llllll}
\hline \multicolumn{2}{l}{ Castelli risk index I } & & & & \\
\hline Variables & & Normal $\mathbf{n}(\%)$ & Changed $\mathbf{n}(\%)$ & Total & p- value \\
\hline \multirow{2}{*}{ Type of diet } & Vegetarian & $54(64.3)$ & $2(16.7)$ & & \\
& Omnivore & $30(35.7)$ & $10(83.3)$ & $40(41.7)$ & $0.003 *$ \\
\hline
\end{tabular}

$* 5 \%$ level of significance $(\mathrm{p}<0.05)$

Table 3 Association between type of diet and percentiles of BDNF plasma levels. São Paulo, Brazil, 2014

\begin{tabular}{|c|c|c|c|c|}
\hline \multicolumn{5}{|c|}{ BDNF plasma levels } \\
\hline Variables & & $<626.0 \mathrm{pg} / \mathrm{ml} \mathrm{n}(\%)$ & $>626.0 \mathrm{pg} / \mathrm{ml} \mathrm{n}(\%)$ & $\begin{array}{l}\text { Chi-Square } \\
\text { Test(p) }\end{array}$ \\
\hline \multirow[b]{2}{*}{ Type of diet } & Vegetarian & $28(60.4)$ & $27(56.3)$ & \\
\hline & Omnivore & $19(39.6)$ & $21(43.8)$ & 0.679 \\
\hline
\end{tabular}

$*_{p}>0.05$. Chi-square test 
Table 4 Plasma levels of BDNF and risk factors for cardiovascular diseases. São Paulo, Brazil, 20 I4

\begin{tabular}{|c|c|c|c|c|}
\hline \multirow[b]{2}{*}{ Variables } & & \multicolumn{3}{|c|}{ Plasma Levels of BDNF } \\
\hline & & $\begin{array}{l}<626.0 \mathrm{pg} / \mathrm{ml} \\
\mathrm{n}(\%)\end{array}$ & $\begin{array}{l}>626.0 \mathrm{pg} / \mathrm{ml} \\
\mathrm{n}(\%)\end{array}$ & Chi-squared test(p) \\
\hline \multirow{2}{*}{$\mathrm{TC}(\mathrm{mg} / \mathrm{dL})$} & Normal & $37(77.1)$ & $4 \mid(85.4)$ & \multirow{2}{*}{1.000} \\
\hline & Altered & II (22.9) & $7(14.6)$ & \\
\hline \multirow{2}{*}{$\mathrm{HDL}(\mathrm{mg} / \mathrm{dL})$} & Normal & $27(56.3)$ & $16(33.3)$ & \multirow{2}{*}{$0.023 *$} \\
\hline & Altered & $21(43.8)$ & $18(37.5)$ & \\
\hline \multirow{2}{*}{ LDL(mg/dL) } & Normal & $40(93.8)$ & $43(89.6)$ & \multirow{2}{*}{0.369} \\
\hline & Altered & $3(6.3)$ & $5(10.4)$ & \\
\hline \multirow{2}{*}{ TGL(mg/dL) } & Normal & $45(97.9)$ & $46(95.8)$ & \multirow{2}{*}{1.000} \\
\hline & Altered & $3(6.3)$ & $2(4.2)$ & \\
\hline \multirow{2}{*}{ WC } & Normal & $43(89.6)$ & $4 \mid(85.4)$ & \multirow{2}{*}{0.302} \\
\hline & Altered & $19(39.6)$ & $21(43.8)$ & \\
\hline
\end{tabular}

$* 5 \%$ level of significance $(p<0.05)$

\section{Discussion}

The present study found that the vegetarian diet is associated with better parameters related to risk factors for cardiovascular diseases, playing, therefore, a protective role in the development of CVD. Furthermore, this study pointed out those subjects with higher concentration of BFNF present lower HDL-c values. Vegetarian subjects had a better nutritional status according to the parameters evaluated, since these are leaner, have lower BMI and lower WC values. In a meta-analysis evaluating 12 randomized clinical trials, they found that the vegetarian diet has a beneficial effect on weight loss. ${ }^{43}$ TONSTAD et al (2009), evaluating 22,434 men and 38,469 women who participated in the Adventist Health Study-2, found that vegans presented a lower BMI mean $(23.6 \mathrm{~kg} / \mathrm{m} 2)$ when compared with omnivores $\left(28.8 \mathrm{~kg} / \mathrm{m}^{2}\right)$, thus indicating an important role of veganism in the protection against obesity. ${ }^{33}$

In addition, central obesity, evaluated through the waist circumference parameter, is an important criterion in the prediction of risk for chronic diseases, especially for cardiovascular diseases ${ }^{34}$ and, in excess, it promotes the release of free fatty acids and triacylglycerols in systemic circulation, which can stimulate the resistance to the action of insulin in peripheral tissues ${ }^{35}$.

In this study, not only WC but also the Castelli Risk Index 1, both cardiovascular risk predictors, were significantly higher in omnivores than in vegetarians, and those who are adept to vegetarian diet increase by 17 times the likelihood of having a normal Castelli Index 1. Rizzo et al., ${ }^{36}$ evaluated the association between a vegetarian diet and the risk for metabolic syndrome in 773 subjects and found results similar to those of this study, in which vegetarians presented significantly lower waist circumference when compared with omnivore subjects ${ }^{36}$. Besides this, the authors found that vegetarian subjects presented a more favorable profile of metabolic risk factors and lower risk for metabolic syndrome and, therefore, lower risk for CVD.

In this sense, in a Brazilian study, in which they evaluated the consumption of nuts and the risk for development of CVD, they administered supplement to 37 obese women, for 8 weeks, with 1 portion of nuts $(290 \mu \mathrm{g}$ of Selenium per day) and, found that supplementation promoted a significant increase in HDL-c concentrations, resulting in a significant improvement of Castelli Risk Indexes 1 and 2, thus reducing cardiovascular risk. ${ }^{37}$ One of the main explanations associated to plant-based diets and a lower risk for CVD consists in the quality of the diet, since it contains foods with a more adequate nutritional value, with whole grains as the main source of carbohydrate, low fat and a better quality (foods rich in monounsaturated and polyunsaturated fats), with an increased consumption of oilseeds, large amount of fruits and vegetables and foods rich in omega-3 fatty acids, which play an important role in the prevention of CVD. ${ }^{38}$

Although there are few Brazilian studies that evaluated the metabolic effects of the vegetarian diet in comparison with the omnivore diet, there is a consensus that the vegetarian diet is associated to lower values of TG, TC and LDL. ${ }^{13,12,39,40}$

Slywitch et al. ${ }^{40}$ evaluated comparatively the metabolic status of vegetarian and omnivore subjects through the blood glucose, lipid and inflammatory profiles, in a cross-sectional study with 59 women aged between 20 and 50 years, with time of diet of more than one year. Subjects were divided into two groups in accordance with the consumption of meat or not. Age and BMI were similar in both groups. The authors also point out that, despite the reduction in TC observed in subjects without meat, there was no difference in relation to the Castelli risk index 1, even though it trended to be lower in the group with no meat. ${ }^{40}$

Still in Brazil, in order to explain the mechanisms by which vegetarians have lower values of plasma lipids, Vinagre et al. ${ }^{14}$ evaluated the plasma kinetics of TG-marked artificial chylomicrons and qualitative aspects of HDL in 39 vegetarians, for at least five years, and 29 omnivores. The results suggest that vegetarian diet accelerates the removal of atherogenic lipoproteins, which can explain the beneficial effect of this diet in relation to the atherosclerotic process. ${ }^{39}$ 
Other methods were also previously proposed in order to test the hypothesis that dietetic factors of vegetarian diet improve sensitivity to insulin and consequently decrease the intramyocellular lipids (IMCL). It concerns a case-control study that evaluated a total of 24 vegans and 25 omnivores. The subjects were paired by gender, age and BMI. The results showed that vegans presented lower fasting triglycerides $(-0.7 \mathrm{mmol} / 1, \mathrm{CI}-0.9$ to $-0.4, \mathrm{P}<0,001)$ and the IMCL levels were significantly lower $(9.7, \mathrm{CI}-16.2$ to $3.3, \mathrm{P}=0.01)$. The authors conclude that vegans have a food intake and biochemical profile that can be cardioprotective, with lower IMCL accumulation. ${ }^{41}$

In a meta-analysis, analyzed 11 randomized clinical studies, examining the effects of vegetarian diets on blood lipid concentrations and suggested that vegetarian diets had a significant reduction effect on the concentrations of TC, LDLc, HDL-c and non-HDL-c in the blood; however, they did not find a significant effect on the concentrations of TG. ${ }^{42}$

Although lipid-lowering drugs like statins and fibrates may be effective in the reduction of concentrations of TC, LDL-C and TG, different health organizations sustain that the modification of dietetic and lifestyle standards is considered an essential approach for the prevention and treatment of dyslipidemia and of cardiovascular disease. $^{42}$ In this sense, correct intake of all food groups may also normalize plasma lipids and lipoproteins and, for this reason, vegetarians are more likely to present desirable parameters of lipemia. In this study, the HDL-c levels were significantly higher and more adequate in vegetarians as compared to omnivores, and it is known that the increased plasma levels of HDL-c decrease the relative risk for CVD. ${ }^{43,44}$

Ashen concluded in a review study that vegetarian diet decreases the likelihood of developing cardiovascular diseases, and it is effective in maintaining desirable values of serum lipids, lowering blood pressure, improving of glycemic control and in the sensitivity to insulin, besides reducing weight. ${ }^{45}$ Data from a meta-analysis, as well as from longitudinal studies with Seventh Day Adventist vegetarians, also show such benefits. ${ }^{46}$

However, it is intriguing to note that the beneficial effects of the vegetarian diet are not observed in interventional studies, when subjects can choose the type of diet for weight $\operatorname{loss}^{47,48}$ although, in a 22-week interventional study with vegan and low-fat diet there was significant reduction of weight and waist circumference measurement ${ }^{49}$ and consequently an improvement of plasma lipid profile.

In a dietary intervention that investigated the effects of the ovolacto-vegetarian diet in clinically healthy omnivorous individuals compared to a 3-month low-calorie Mediterranean diet with a crossdesign in 118 subjects, it was found that both a diet vegetarian as well as mediterranean were effective in reducing body weight, body mass index and fat mass without significant differences between them. However, the vegetarian diet was more effective in lowering cholesterol levels of low density lipoproteins. ${ }^{50}$

As major clinical implications related to vegetarian diet outcomes, the reduction of risk factors associated with the development of chronic diseases. Chiu et al (2018) investigated an association between vegetarian diet, changing patterns and dietary risk of diabetes in a Buddhist $(\mathrm{n}=2918)$ Taiwanese population. Results obtained from the vegetarian diet were associated with $35 \%$ lower risks (HR: 0.65 , $95 \%$ CI: $0.46,0.92$ ), while converting a non-vegetarian standard to vegetarians was associated with 53\% risks (HR: $0.47,95 \%$ CI: 0.30 , 0.71 ) for diabetes compared to non-vegetarians, thus suggesting that vegetarian diet and conversion to vegetarian diet may protect against diabetes used by BMI among Taiwanese. ${ }^{51}$

Also, as chronic diseases such as obesity, metabolic syndrome, involve common features like inflammatory processes. In this sense, Haghighatdoost et al. ${ }^{52}$ point out that vegetarianism is associated with changes in lower C-reactive protein (CRP) levels when students use a vegetarian diet for less than 2 years. Different studies $(6,10,11,13)$ have documented the important role of vegetarian diets in lipid profile modulation (decreased LDL-c, TC, TG), increased HDL-c, with clinical implications related to the prevention of chronic diseases, especially cardiovascular disease and decreased risk of mortality. ${ }^{52}$

It is also important to emphasize that the pathogenesis of atherosclerosis is a complex process, and that the cardiovascular protective effects of the vegetarian diet are multifactorial. Therefore, the risk factors should be judged together, instead of individually. This way, the benefits of reducing serum cholesterol levels are not necessarily translated into a reduced risk for cardiovascular diseases in this population. For this reason, it is important to evaluate other biomolecular pathways that may explain the effect of the vegetarian diet in the prevention of cardiovascular disease.

Thus, evaluating molecular parameters that may explain, through other ways, the benefit of the vegetarian diet in cardiovascular health is necessary. Regarding BDNF, vegetarians presented the lowest means, but, in this study, there was no difference between plasma BDNF means between vegetarians and omnivores. The BDNF plasma values found here are similar to the results of Karczewska-Kupczewska et al., ${ }^{29}$ who evaluated a similar population in terms to age, BMI and gender distribution.

Jawale et al., ${ }^{53}$ evaluating the effect of milk fat consumption during pregnancy in an animal model, specifically in relation to fatty acid parameters, brain neurotrophins (brain-derived neurotrophic factor: BDNF; and nerve growth factor: NGF). ) and cognitive performance in adult offspring identified that adult offspring cognitive performance of various food groups (hyperlipid diet; high fat diet supplemented with omega-3 fatty acids; high fat diet, vitamin B12 deficient; high fat deficiency in vitamin B12 supplemented with omega-3 fatty acids) was similar to the control. In conclusion, consumption of a high-fat dairy maternal diet, while reducing the levels of cerebral BDNF in the pup at birth, does not affect the cognitive health of adult offspring. ${ }^{53}$

In the same direction, Perrin et al..$^{54}$ evaluated the association between supplement use patterns and brain-derived neurotrophic factor (BDNF) fatty acid concentrations in breast milk of women following vegan, vegetarian and omnivorous dietary patterns. and found that the breast milk of vegan women had significantly more unsaturated fats and total omega- 3 fats, and fewer saturated fats, trans and omega- 6 to omega-3 fats than omnivorous women, however, BDNF did not was detectable in any sample. ${ }^{54}$

After analysis of associations by percentiles of BDNF, using as cut-off point its median, it was found that the higher the concentration of BDNF, the higher was the risk of having an altered HDL-c (52\%). ZEMBRON-LACNY et al. (2016), studying the changes related to age in peripheral BDNF and its relation with markers of oxidative stress in active and inactive men, found an inverse correlation between BDNF and risk factors for cardiovascular diseases (Castelli Risk Index 1, 
C-reactive protein and oxidized LDL). ${ }^{55}$ If the BDNF contributes to the pathogenesis of CVD's or, if the change in the levels of this factor occurred as an adaptive response to cellular stress, the data found do not permit it to respond with accuracy. It is known that plasma BDNF is associated to risk factors for CVD's and metabolic syndrome (MS); however, the platelet levels can also provide some explanations. First, because these figurative elements of the blood store large amounts of BDNF and may be altered in obese patients, since TC and TG could harm this storage. ${ }^{26}$

Sustar et al (2019) evaluated whether plasma BDNF concentration was associated with coronary heart disease in ethnically homogeneous groups of patients and correlate plasma BDNF levels with known risk factors for coronary heart disease. Plasma BDNF concentration was significantly $(\mathrm{P}<0.01)$ reduced in patients with coronary heart disease compared to controls, therefore, the results revealed lower plasma BDNF concentration in patients with coronary heart disease, suggesting that a decrease in plasma BDNF concentration BDNF may be associated with the pathogenesis of coronary heart disease. ${ }^{56}$

Although there may be significant evidence, the effects of BDNF on the cardiovascular system are still not well understood. BDNF, as a neurotrophin, acts on the tyrosine kinase $\mathrm{B}$ (Trk-B) receptor present in the CNS, but can also cross the blood-brain barrier and become circulating. Plasma BDNF can then induce or inhibit pathways outside the central nervous system ${ }^{57}$ and, for this reason, it can be involved in the vulnerability of the atherosclerosis plaque. ${ }^{58}$ Studies in animals have demonstrated that BDNF and the tyrosine kinase B (Trk-B) receptor play an important role in the development of the cardiovascular system. ${ }^{59}$

BDNF can lead to platelet instability in the atherosclerotic plaque through the ability of inducing oxidative stress and promoting the generation of superoxide radicals. Besides this, BDNF has shown to induce oxidative stress through activation of the $\mathrm{NAD}(\mathrm{P}) \mathrm{H}$ oxidase system in the cardiovascular system. ${ }^{59}$ It is also known that the central nervous system is sensitive to oxidative stress and that BDNF can exert a protective effect under conditions of DNA damage. ${ }^{60-62}$

Eriji et al. showed that the plasma BDNF levels are increased in coronary circulation of subjects with diagnosis of stable angina, as well as the BDNF expression in coronary arteries of subjects of this group. BDNF also increases the activity of NAD (P)H oxidase and the production of peroxides in human vascular cell cultures. This way, the authors concluded that the increase of oxidative stress induced by BDNF has an important action in the stability of the atheroma plaque..$^{58}$

For this reason, it is believed that the inverse relation between the BDNF and HDL-c levels found in this study may represent a response to oxidative stress caused by the decrease of HDL-c, in those with these altered parameters. In this sense, it is worth noting the importance of new studies investigating the role of BDNF in cardiovascular diseases, by evaluating inflammatory and oxidative stress parameters.

However, some limitations of this study must be considered. Primarily, because it is a cross-sectional study, in which subjects are evaluated only once. In addition to being a small population and a convenience sample (as described in the methodology), they were evaluated at a single research center (Paulista University, São Paulo, Brazil). Inflammatory and cardiorespiratory capacity (such as maximum VO2) parameters were not evaluated, which can be related with changes in the plasma concentrations of BDNF, and data on the subjects' food consumption were not evaluated as well. This way, a new research agenda is opened, seeking to link the relationship between BDNF and cardiovascular risk, taking into consideration such aspects.

\section{Conclusion}

The data found in this study point out that the vegetarian diet is associated with better parameters related to risk factors for cardiovascular diseases, thus playing a protective role in the development of CVD. In relation to the BDNF expression and the standard of diet, statistically significant results were not found. The highest BDNF concentration in subjects with lower HDL-c values can be attributed to an adaptative response to the oxidative stress.

\section{Acknowledgments}

Our deepest gratitude to all research volunteers, for the valuable contribution. We are also grateful for the financial support granted by the Fundação de Apoio à Pesquisa Research Support Foundation (FAPESP, process No. 2013/11057-3 and process No. 2016/08758-8) and for the contribution of Rosana Camarini and André Veloso Lima Rueda for the help in laboratory analysis.

\section{Conflicts of interest}

The authors declare no conflict of interest. The founding sponsors had no role in the design of the study; in the collection, analyses, or interpretation of data; in the writing of the manuscript, and in the decision to publish the results.

\section{References}

1. Ministry of Health. Health Surveillance Secretariat. Health Situation Analysis Department. Strategic action plan for the fight against chronic noncommunicable diseases (NCDs) in Brazil 2011-2022. Brazil: Ministry of Health; 2011. p. 1-160.

2. Mansur AP, Favarato D. Mortality from cardiovascular diseases in Brazil and the metropolitan region of São Paulo: 2011 update. Arq Bras Cardiol. 2012;99(2):755-761.

3. Ministry of Health. Health Brazil 2014: an analysis of the health situation and external causes. Health Surveillance Secretariat. Department of Noncommunicable Diseases Surveillance and Health Promotion. Brazil: Ministry of Health; 2015. p. 1-130.

4. Libby P. Inflammation in atherosclerosis. Nature. 2002;420(6917):868874.

5. Ministry of Health. Health: surveillance of risk factors and protection for chronic diseases by telephone survey [electronic resource]. Brazil: Ministry of Health; 2017. p.

6. Hu FB. Plant-Based foods and prevention of cardiovascular disease: an overview. Am J ClinNutr. 2003;78(3 Suppl):544S-551S

7. Key TJ, Appleby PN, Spencer EA, et al. Cancer incidence in British vegetarians. Br J Cancer. 2009;101(1):192-197.

8. Craig WJ, Mangels AR; American Dietetic Association. Position of the American Dietetic Association: Vegetarian diets. J Am Diet Assoc. 2009;109(7):1266-1282.

9. Orlich MJ, Fraser GE. Vegetarian diets in the Adventist Health Study 2: a review of initial published findings. Am J Clin Nutr. 100 Suppl 1:353S$8 \mathrm{~S}$ 
10. Fraser GE. Vegetarian diets: what do we know of their effects on common chronic diseases? Am J Clin Nutr. 2009;89(5):1607S-1612S.

11. Orlich MJ, Singh PN, Sabaté J, et al. Vegetarian dietary patterns and mortality in Adventist Health Study 2. JAMA Intern Med. 2013;173(13):1230-1238.

12. De Biase SG, Fernandes SFC, Gianini RJ, et al. Vegetarian Diet and Cholesterol and Triglyceride Levels. Arq Bras Cardiol. 2007;88(1):3539.

13. Teixeira Rde C, Molina Mdel C, Zandonade E, et al. Cardiovascular Risk in Vegetarian and Omnivores: A Comparative Study. Arq Bras Cardiol. 2007;89(4):237-244.

14. Vinagre JCM. Effects of vegetarian diet on chylomicron metabolism and qualitative aspects of high density protein (HDL) [Doctoral Thesis]. São Paulo: USP School of Medicine; 2010.

15. Jacob RA, Burri BJ. Oxidative damage and defense. Am J Clin Nutr. 1996;63(6):985S-990S.

16. Messina MJ, Messina VL. The dietitian's Guide to Vegetarians Diets: Issue and Applications. Gaithersburg, MD: Aspen Publishers; 1996.

17. Farmer B, Larson BT, Fulgoni VL 3rd, et al. A vegetarian dietary pattern as a nutrient-dense approach to weight management: an analysis of the national health and nutrition examination survey 1999-2004. J Am Diet Assoc. 2011;111(6):819-827.

18. Neeper SA, Gómez-Pinilla F, Choi J, et al. Exercise and brain neurotrophins. Nature. 1995;373(6510):109.

19. Mattson MP, Maudsley S, Martin B. BDNF and 5-HT: a dynamic duo in age- related neuronal plasticity and neurodegenerative disorders. Trends Neurosci. 2004;27(10):589-594.

20. Lebrun B, Bariohay B, Moyse E, et al. Brain-derived neurotrophic factor (BDNF) and food intake regulation: a minireview. Auton Neurosci. 2006;126-127:30-38.

21. Tyler WJ, Alonso $\mathrm{M}$, Bramham $\mathrm{CR}$, et al. From acquisition to consolidation: on the role of brain-derived neurotrophic factor signaling in hippocampal- dependent learning. Learn Mem. 2002;9(5):224-237.

22. Jung SH, Kim J, Davis JM, et al. Association among basal serum BDNF, cardiorespiratory fitness and cardiovascular disease risk factors in untrained healthy Korean men. Eur J Appl Physiol. 2011;111(2):303-311.

23. Jiang H, Liu Y, Zhang Y, et al. Association of plasma brain-derived neurotrophic factor and cardiovascular risk factors and prognosis in angina pectoris. Biochem Biophys Res Commun. 2011;415(1):99-103.

24. Swardfager W, Herrmann N, Marzolini S, et al. Brain derived neurotrophic factor, cardiopulmonary fitness and cognition in patients with coronary artery disease. Brain Behav Immun. 2011;25(6):1264-1271.

25. Suwa M, Kishimoto H, Nofuji Y, et al. Serum brain-derived neurotrophic factor level is increased and associated with obesity in newly diagnosed female patients with type 2 diabetes mellitus. Metabolism. 2006;55(7):852-857.

26. Krabbe KS, Nielsen AR, Krogh-Madsen R, et al. Brain-derivaded neurotorphic factor (BDNF) and type 2 diabetes. Diabetologia. 2007;50(2):431-438

27. Lorgis L, Amoureux S, de Maistre E, et al. Serum brain-derived neurotrophic factor and platelet activation evaluated by soluble $\mathrm{P}-$ selectin and soluble CD-40-ligand in patients with acute myocardial infarction. Fundam Clin Pharmacol. 2010;24(4):525-530.

28. Sánchez-Villegas A, Galbete C, Martinez-González MA, et al. The effect of the Mediterranean diet on plasma brain-derived neurotrophic factor (BDNF) levels: The PREDIMED-NAVARRA randomized trial. Nutr Neurosci. 2011;14(5):195-201.
29. Karczewska-Kupczewska M1, Strączkowski M, Adamska A, et al. Decreased serum brain-derived neurotrophic factor concentration in young nonobese subjects with low insulin sensitivity. Clin Biochem. 2011;44(10-11):817-820.

30. World Health Organization. Phisycal Status: the use and interpretation of anthropometry. Geneva, Switzerland: World Health Organization; 1995.

31. World Health Organization. Obesity: Preveting and Managing the Global Epidemic. Geneva, Switzerland: World Health Organization; 1998.

32. Huang RY, Huang CC, Hu FB, et al. Vegetarian diets and weight reduction: A meta-analysis of randomized controlled trials. J Gen Intern Med. 2016;31(1):109-116.

33. Tonstad S, Butler T, Yan R, et al. Type of vegetarian diet, body weight, and prevalence of type 2 diabetes. Diabetes Care. 2009;32(5):791-796.

34. Lima CG De, Basile LG, Silveira JQ Da, et al. Circunferência da cintura ou abdominal? Uma revisão crítica dos referenciais metodológicos. Rev Simbio-Logias. 2011;4(6):108-131.

35. Lunardi CC, Petroski EL. Body mass index, waist circumference and triceps skinfold in predicting lipid changes in 11-year-old children. Arq Bras Endocrinol Metab. 2008;52(6):1009-1014

36. Rizzo NS, Sabaté J, Jaceldo-Siegl K, et al. Vegetarian Dietary Patterns Are Associated With a Lower Risk of Metabolic Syndrome: The Adventist Health Study 2. Diabetes Care. 2011;34(5):1225-1227.

37. Cominetti C, de Bortoli MC, Garrido AB Jr, et al. Brazilian nut consumption improves selenium status and glutathione peroxidase activity and reduces atherogenic risk in obese women. Nutr Res. 2012;32(6):403-407.

38. Hu FB. Plant-Based foods and prevention of cardiovascular disease: an overview. Am J ClinNutr. 2003;78(3 Suppl):544-551.

39. Vinagre JC, Vinagre CG, Pozzi FS, et al. Metabolism of triglyceriderich lipoproteins and transfer of lipids to high-density lipoproteins (HDL) in vegan and omnivore subjects. Nutr Metab Cardiovasc Dis. 2013;23(1):61-67.

40. Slywitch E, Nóbrega FJ D, Duarte ACG. Assessment of metabolic and nutritional status of vegetarian and omnivorous individuals. Rev Bras Nutr Clin. 2012;27:80-86.

41. Goff LM, Bell JD, So PW, et al. Veganism and its relationship with insulin resistance and intramyocellular lipid. Eur J Clin Nutr. 2005;59(2):291298.

42. Wang F, Zheng J, Yang B, et al. Effects of Vegetarian Diets on Blood Lipids: A Systematic Review and Meta-Analysis of Randomized Controlled Trials. J Am Heart Assoc. 2015;4(10):e002408.

43. Ahima RS, Flier JS. Adipose tissue as naendocrine organ. Trends Endocrinol Metab. 2000;11(8):327-332.

44. World Health Organization. Integrated Prevention of Noncommunicable Diseases. Geneva, Switzerland: World Health Organization; 2003.

45. Dominique Ashen M. Vegetarian diets in cardiovascular prevention. Curr Treat Options Cardiovasc Med. 2013;15(6):735-745.

46. Kwok CS, Umar S, Myint PK, et al. Vegetarian diet, Seventh Day Adventists and risk of cardiovascular mortality: a systematic review and meta-analysis. Int J Cardiol. 2014;176(3):680-686.

47. Burke LE, Hudson AG, Warziski MT, et al. Effects of a vegetarian diet and treatment preference on biochemical and dietary variables in overweight and obese adults: a randomized clinical trial. Am J Clin Nutr. 2007;86(3):588-596.

48. Burke LE1, Steenkiste A, Music E, et al. A Descriptive Study of Past Experiences with Weight-Loss Treatment. J Am Diet Assoc. 2008;108(4):640-647. 
49. Ferdowsian HR, Barnard ND, Hoover VJ, et al. A multicomponent intervention reduces body weight and cardiovascular risk at a GEICO corporate site. Am J Health Promot. 2010;24(6):384-387.

50. Sofi F, Dinu M, Pagliai G, et al. Low-Calorie Vegetarian Versus Mediterranean Diets for Reducing Body Weight and Improving Cardiovascular Risk Profile: CARDIVEG Study (Cardiovascular Prevention With Vegetarian Diet). Circulation. 2018;137(11):1103-1113.

51. Chiu THT, Pan WH, Lin MN, Vegetarian diet, change in dietary patterns, and diabetes risk: a prospective study. Nutr Diabetes. 2018;8(1):12.

52. Haghighatdoost F, Bellissimo N, Totosy de Zepetnek JO, et al. Association of vegetarian diet with inflammatory biomarkers: a systematic review and meta-analysis of observational studies. Public Health Nutr. 2017;20(15):2713-2721.

53. Jawale S, Joshi S, Kale A. Maternal dairy fat diet does not influence neurotrophin levels and cognitive performance in the rat offspring at adult age. Int J Dev Neurosci. 2018;71:18-29.

54. Perrin MT, Pawlak R, Dean LL, et al. A cross-sectional study of fatty acids and brain-derived neurotrophic factor (BDNF) in human milk from lactating women following vegan, vegetarian, and omnivore diets. Eur J Nutr. 2019;58(6):2401-2410.

55. Zembron-Lacny A, Dziubek W, Rynkiewicz M, et al. Peripheral brainderived neurotrophic factor is related to cardiovascular risk factors in active and inactive elderly men. Braz J Med Biol Res. 2016;49(7).
56. Sustar A, Perkovic MN, Erjavec GN, et al. Association between reduced brain-derived neurotrophic factor concentration \& coronary heart disease. Indian J Med Res. 2019;150(1):43-49.

57. Rosas-Vargas H, Martínez-Ezquerro JD, Bienvenu T. Brain-derived neurotrophic factor, food intake regulation, and obesity. Arch Med Res. 2011;42(6):482-494.

58. Ejiri J, Inoue N, Kobayashi S, et al. Possible Role of Brain-Derived Neurotrophic Factor in the Pathogenesis of Coronary Artery Disease. Circulation. 2005;112(14):2114-2120.

59. Noren Hooten N, Ejiogu N, Zonderman AB, et al. Protective Effects of BDNF against C-Reactive Protein-Induced Inflammation in Women. Mediators Inflamm. 2015;2015:516783.

60. Russo-Neustadt, A. (2003) Brain-derived neurotrophic factor, behavior, and new directions for the treatment of mental disorders. Semin Clin Neuropsychiatry. 2003;8(2):109-118.

61. Bathina S, Das UN. Brain-derived neurotrophic factor and its clinical implications. Arch Med Sci. 2015;11(6):1164-1178.

62. Chen SD, Wu CL, Hwang WC, Y More Insight into BDNF against Neurodegeneration: Anti-Apoptosis, Anti-Oxidation, and Suppression of Autophagy. Int J Mol Sci. 2017;18(3). pii: E545. 